\title{
Elements of learning technologies designing of engineering networks heat
}

\author{
Irina G. Sidorkina ${ }^{1, *}$ and Oleg L. Sorokin ${ }^{1}$ \\ ${ }^{1}$ Volga State University of Technology, 424000, Yoshkar-Ola, Russia
}

\begin{abstract}
Modern educational systems function as a medium fast analysis of shared information that defines them as analytical. The purpose of analytical information processing systems: working with distributed data on a global computer networks, mining and processing of semi structured information, knowledge. Existing mathematical and heuristic methods for the automated synthesis of electronic courses and their corresponding algorithms do not allow the full compliance of development realized in the form of adequate criteria for the totality of the properties distributed educational systems within acceptable time limits and characteristic. Therefore, the development of electronic educational applications must be accompanied by a variety of software support intelligent and adaptive functions. In addition, there is no theoretical justification for integrative aspects and their practical applications for intelligent and adaptive systems of designing distance learning courses. Currently, this type of problem may be considered as a potentially promising. The article presents the functionality of the e-learning course on the design engineering of thermal networks, process modeling in engineering networks with the solution of energy efficiency, detection of problem areas; identify the irrational layout of heaters and others.
\end{abstract}

\section{Introduction}

The development of information and communication technologies and means of communication has defined the main trends in the organization of training courses for engineering design of heat networks associated with providing remote access to design the project and the intellectualization of training aids. The electronic distance learning course is carried out through the basics of Thermal Physics design type for a given embodiment of the circuit. Intelligent CAD components, as well as the Multi Media Interface (MI) allow providing information in a convenient and informative to read format. In addition, a set of recommendations identifies errors in the construction of thermal circuit (TC) network engineering.

MI electronic course implemented based on the identification situations algorithms predict their development in real time, timely and relevant synthesis descriptions of system behavior in the various cases. This allows the student to give timely advice and to exclude nonoptimal solutions. In addition, Mi algorithms designed for imaging heat flow prediction [1] freezing the building structure (Fig. 1), heater layout, detection of problem areas in the IP, as well as identify patterns in raw data about temperature indicators, implement problem-solving-based technology examples. Features of the application of visualization elements of the course, is the use of the color gradation of heat flows in the reporting calendar period (shown in Figure 2). In the case of overlay heat flows from the heater and result visualization utilities will view the resulting stream, the temperature of which corresponds to the actual temperature in the projected or reconstructed building. The proposed colors are optimal for perception and use all the gradations of color in the transition from cold to warm heat flow.

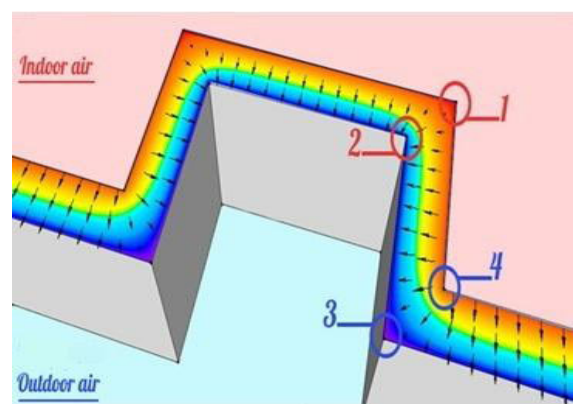

Fig.1. The freezing of the building contour, where 1.2 the problem areas of the internal angle and 3.4 external

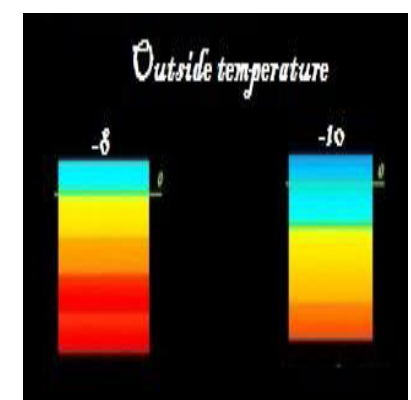

Fig.2. Examples of color-imaging in the e-learning environment for engineering design of heat networks 
The technology of decision support tasks, based on the examples, which is the latest technology. It helps students to solve new problems without formulating their mistakes, but offering has successfully solved the problem, from an earlier experience.

In the field of traditional applications, interactive problem solving support is dominant over the other technologies.

\section{Intelligent algorithms training arrangement TC CAD}

Adaptive Hypermedia technology so implement custom models to adapt the content of pages and links to the user. The task of intelligent components [2] e-learning course on CAD thermal utilities, to provide the most optimal variant TC Layout, trace a connection point with the heat, so as to bring the course content of the page as possible to the user. The result of the algorithm is to visualize the nature of heat flow for a specific situation, taking into account the algorithm of identification of situations and decision-making.

An analysis of the proposed definitions for intelligent CAD information model [3] shows that it displays its own dynamics of the design process, ie, sequential process recreating all necessary technical specification of design characteristics of the object (TC), and creates a fragmentary compilation in its description and provides transfer of data to knowledge.

The input data rate to the user in this case are:

Generalized objective function of the adaptive module and a set of functions for visualization.

-Information Model of adaptive module for visualization of heat fluxes, built on the basis of the totality of the measurements with the laboratory setup.

- Overlays flow (from heaters, of walling)

Laws covering the logic and methods for detection and identification of thermal circuit situations.

The result of the processing of the measuring information received from multiple sensors - recognition options situations subsequent trace heaters. Hierarchy overlay flows represents a fusion of the two fronts warm from the heaters and the cold of the contour. The result is the total heat flow, which is then used for making decisions about repackaging heaters. Identifying situations thermal circuit eventually has only two options: no problem zones (zones freezing), or their availability.

We define the basic steps of the algorithm identifying situations and the decision implemented in the education system of the design engineering of thermal networks:

Step 1: On the basis of the generated priori constraint matrix using a linear programming technique forming confidence intervals.

Step 2. Form matrix circuit restrictions, defining the boundaries of the impact of the heat flow.

Step 3: Checking if the matrix is "code-decision" on the existence of an adequate solution.

Step 4. If a solution is found, then the operation code decides on imaging heat flow in a certain way.
Step 5. If the solution is not found or there are several solutions (fuzzy situation), then using a probability matrix is a solution with maximum probability.

Step 6. If the survey matrix "code-decision" is not ambiguous situation, on a binary code of relations of memory values, the most appropriate sector frames [4], which followed on the criterion of self-identified the most suitable situation.

Thus, the selection function is implemented by the new situation of choice (code binary operations) most suitable frames sector in the knowledge base. [4]

The following are examples of rules-based system of training knowledge engineering design of heat networks in e-learning environment:

Rule1. If the coordinates of the heat distribution of the heater flow and heat flux coordinate engineering network circuit are THEN calculate the resulting heat flow.

Rule2. If the resulting heat flows at a temperature of less than optimal, we find the area of the problem area.

Rule 3: If I find a problem area of the zone, the minimal vector construct to the contour of utilities to locate the problem area.

Rule 4: If I find a problem area or problem area is issued a certificate of non-optimal arrangement and performing layout again.

Recognition of the situation is necessary for the possibility of setting temperature environment outside the contour, as well as granting councils in the case where the heat flow from the heater can heat the entire circuit. Elements of Learning Technologies Engineering Design of heating networks should be offered in this case, the best option arrangement, to perform communication with a heat tracing point and visualize the resulting heat flow from the heaters in the building loop. The final stage of the work is to visualize the nature of heat flow for a specific situation, taking into account the algorithm of identification of situations and decision-making adaptive presentation.

The goal of adaptive presentation technology is to adapt the content of a hypermedia page to the objectives, knowledge and other information stored in the user model. In a system with adaptive presentation, the pages are not static, but adaptively generated or assembled from pieces for each user. For example, expert users receive more detailed and in-depth information, while novices receive more additional explanation.

Using fuzzy logic and methods for the synthesis and identification of situations in the electronic course, possible to apply the algorithm of identification of situations and decision-making, based on adaptive representation of information that takes into account all the possible outcomes in the data visualization. Using complex algorithms, the data will allow the most accurate visualization of heat flows in multimidedia $\mathrm{CAD}$ interface $\mathrm{OK}$, which will increase the accuracy of calculations and impact in the future on the quality of the layout of the heating elements in the design of the circuit.

Example visualization recommendations when selfcontrol electronic course participant in the arrangement 
of the heaters is presented in Figure 3.A set of additional features implemented in the course is aimed primarily at expanding functionality and increasing applicability of learning technologies for a number of minor problems associated with additional calculations, advice, construction of all kinds depending graphs, which can be used for visualization of thermal dependencies, for further detailed analysis in the practical work, as well as to implement additional self-monitoring capabilities.
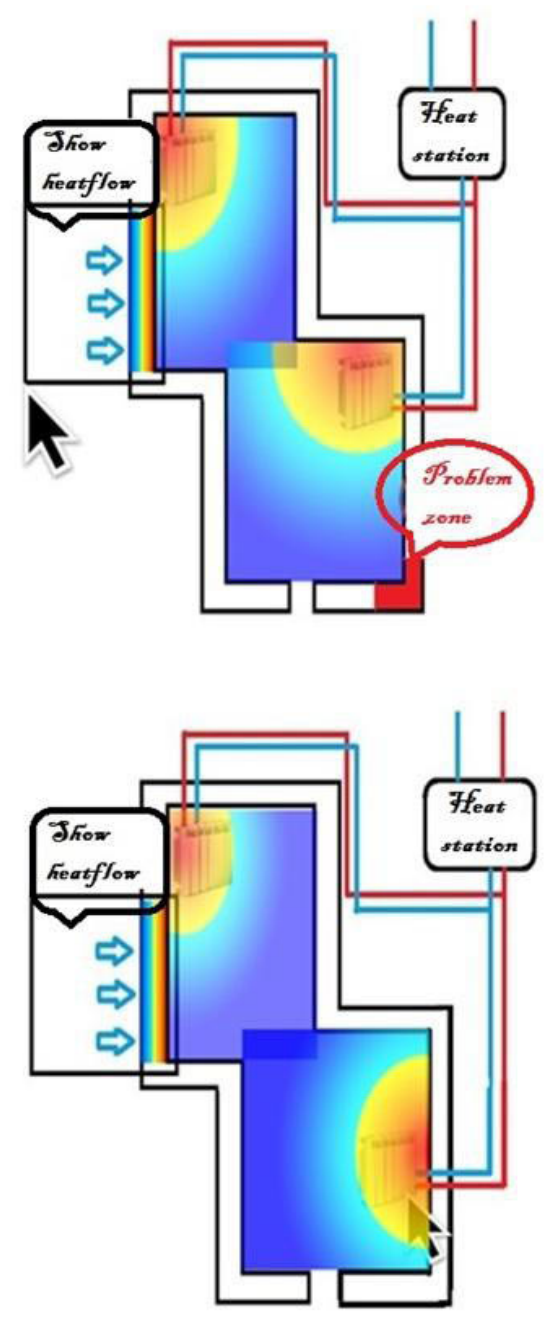

Fig. 3. Visualization of recommendations for the user of the course in solving the layout problem

An example of realized visualization of dependencies may be depending on the degree of freezing of the circuit on the ambient temperature, the impact of surges in ambient temperature on the heat flow visualized and others.

Figure 4. It shows an example of visualization of full contour freezing time depending on the ambient temperature surges. The use of this additional functionality will improve the perception of the theoretical material by diverting the course participant on the graphic data.

Simulation of various processes, such as heaters malfunction and the time necessary user reaction to possible malfunctions in the heating system will allow perceiving the situation more correctly.
Adding more interactivity in educational e-learning modules will increase the involvement of participants in the educational process and remote interest in the tracking of dependencies.

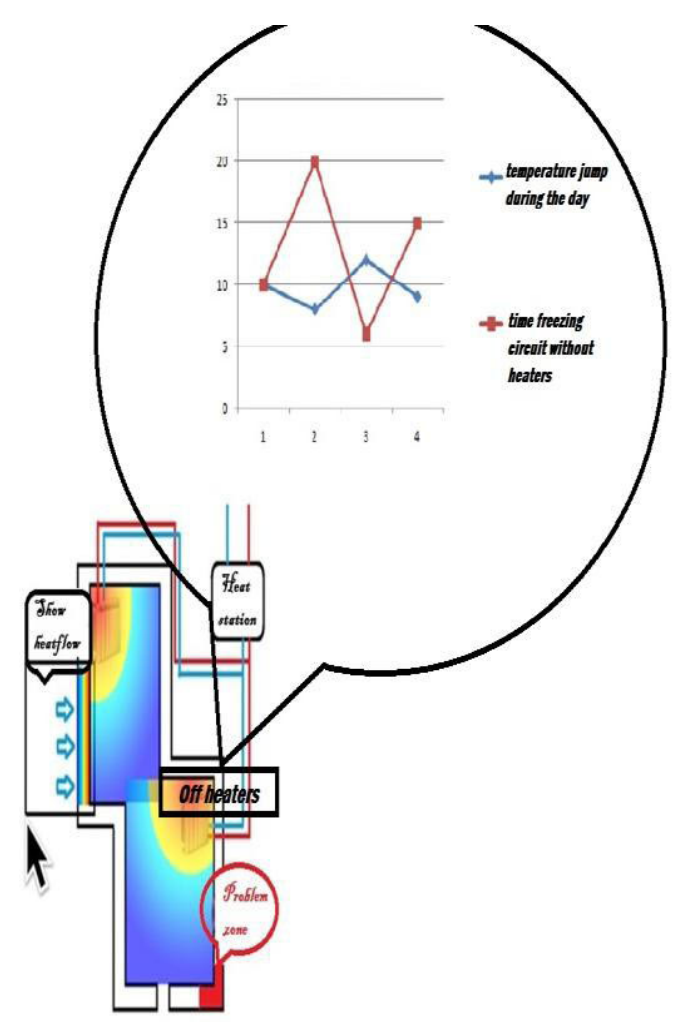

Fig. 4. The character changes depending on the time of freezing circuit when turned off heaters from strong shocks ambient temperature.

One of the main conditions for the development of information-informative independence of students of the University is a network of educational interaction.

Let's determine the definitions that are important for this work: «network», «educational network», «network cooperation», «network pedagogical impact», «network sectorial educational program».

Involving the sources of potential information, the student forms personal (individual) education network PLN (personal learning network). The learning is doe through many channels as different relations are used to deliver educational material. Development of informative and cognitive independence is one of the key pedagogical tasks for modern education system as the ability to learn something new I more important than current knowledge that we possess (ability to enrich knowledge is more important than the static system of accumulated knowledge) [10].

\section{Conclusions}

Application for the development of e-learning components and elements of intelligent MI imaging with the use of adaptive technology and technology solving practical problems, for the task of training the 
engineering design of heat networks, improves the feedback to inform the user of possible random errors. You should also note the presence of elements of selfcontrol. Data Visualization in the calculation reduces the error in the calculations and improves the visual results, as well as to provide support for decision-making in the use of building automation systems solutions.

The proposed approach to the modeling of the navigation elements and the course is consistent with the international specifications of e-learning SCORM and IMS, complementing their other adaptive algorithms to navigate through training courses through the use of integrated assessment model student's knowledge [5].

\section{References}

1. A.J. Nomerchuk, Engineering SFU-C, 2, 56-60 (2013)
2. O.L. Sorokin, I.G. Sidorkina, The module definition of a stationary regime in CAD external engineering networks «IS \& IT-Intelligent CAD 2015" Labour Congress on Intelligent Systems and Information Technology, p. 70-75 (Taganrog, 2015)

3. O.V. Koshkin, I.G. Sidorkina, Open intelligent CAD technology training systems, IEEE International Conference on Systems of artificial intelligence, p. 418-421 (Divnomorskoye, 2002)

4. I.N. Vdovichenko Features create a knowledge base of standard examination techniques of advanced technologies Magazine, 6, 13-16 (2010)

5. V.I. Anisimov, I.G. Sidorkina, Base-aided design techniques training course. Proceedings of ETU "LETI", Ser. "Computer science, management and computer technology", 1, 3-4 (2002)

6. I.V. Robert, Modern information technologies in education: didactic problems; prospects of application (1994) 